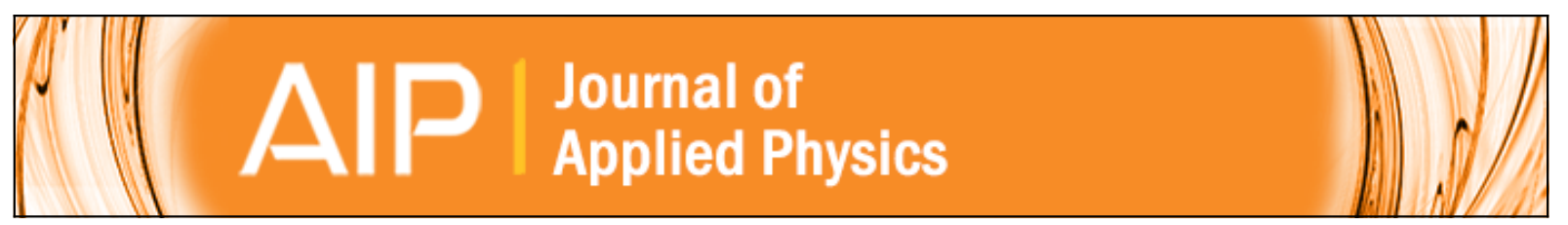

\title{
Shock induced damage in copper: A before and after, three-dimensional study
}

David B. Menasche, Jonathan Lind, Shiu Fai Li, Peter Kenesei, John F. Bingert, Ulrich Lienert, and Robert M. Suter

Citation: Journal of Applied Physics 119, 154902 (2016); doi: 10.1063/1.4947270

View online: http://dx.doi.org/10.1063/1.4947270

View Table of Contents: http://scitation.aip.org/content/aip/journal/jap/119/15?ver=pdfcov

Published by the AIP Publishing

\section{Articles you may be interested in}

Molecular dynamics studies of thermal dissipation during shock induced spalling

J. Appl. Phys. 114, 123509 (2013); 10.1063/1.4821341

Microstructure effects on shock response of Cu nanofoams

J. Appl. Phys. 114, 073501 (2013); 10.1063/1.4818487

Shock-induced consolidation and spallation of Cu nanopowders

J. Appl. Phys. 111, 013508 (2012); 10.1063/1.3675174

Three-dimensional grain mapping by $\mathrm{x}$-ray diffraction contrast tomography and the use of Friedel pairs in diffraction data analysis

Rev. Sci. Instrum. 80, 033905 (2009); 10.1063/1.3100200

Electromigration in passivated $\mathrm{Cu}$ interconnects studied by transmission $\mathrm{x}$-ray microscopy

J. Vac. Sci. Technol. B 20, 3089 (2002); 10.1116/1.1523403

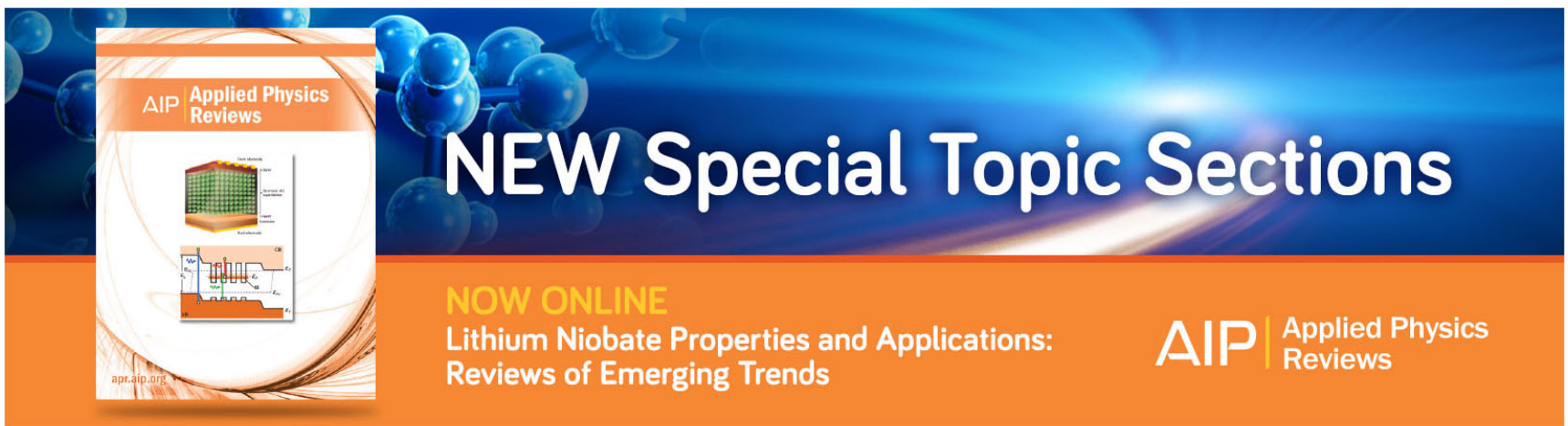




\title{
Shock induced damage in copper: A before and after, three-dimensional study
}

\author{
David B. Menasche, ${ }^{1, a)}$ Jonathan Lind, ${ }^{2}$ Shiu Fai Li ${ }^{3}$ Peter Kenesei, ${ }^{4}$ John F. Bingert, ${ }^{5}$ \\ Ulrich Lienert, ${ }^{6}$ and Robert M. Suter ${ }^{1}$ \\ ${ }^{1}$ Physics Department, Carnegie Mellon University, Pittsburgh, Pennsylvania 15213, USA \\ ${ }^{2}$ Materials Engineering Division, Lawrence Livermore National Laboratory (LLNL), Livermore, \\ California 94550, USA \\ ${ }^{3}$ Computational Engineering Division, LLNL, Livermore, California 94550, USA \\ ${ }^{4} X$-ray Science Division, Argonne National Laboratory, Argonne, Illinois 60439, USA \\ ${ }^{5}$ Los Alamos National Laboratory, Los Alamos, New Mexico, 87544, USA \\ ${ }^{6}$ Deutsches Elektronen-Synchrotron, Hamburg, Germany
}

(Received 9 December 2015; accepted 10 April 2016; published online 21 April 2016)

\begin{abstract}
We report on the microstructural features associated with the formation of incipient spall and damage in a fully recrystallized, high purity copper sample. Before and after ballistic shock loading, approximately $0.8 \mathrm{~mm}^{3}$ of the sample's crystal lattice orientation field is mapped using non-destructive near-field High Energy Diffraction Microscopy. Absorption contrast tomography is used to image voids after loading. This non-destructive interrogation of damage initiation allows for novel characterization of spall points $v i s-a$-vis microstructural features and a fully 3D examination of microstructural topology and its influence on incipient damage. The spalled region is registered with and mapped back onto the pre-shock orientation field. As expected, the great majority of voids occur at grain boundaries and higher order microstructural features; however, we find no statistical preference for particular grain boundary types. The damaged region contains a large volume of $\Sigma-3\left(60^{\circ}\langle 111\rangle\right)$ connected domains with a large area fraction of incoherent $\Sigma-3$ boundaries. Published by AIP Publishing. [http://dx.doi.org/10.1063/1.4947270]
\end{abstract}

\section{INTRODUCTION}

Understanding the plastic response of materials has been a longstanding goal for scientists who desire to engineer them for specific applications. ${ }^{1-3}$ Recent advances in nondestructive characterization have allowed for some of the first direct comparisons between quasi-static models of plasticity in polycrystals ${ }^{4-7}$ and experiment. ${ }^{8-10}$ With the goal of linking to dynamic models, ${ }^{11-15}$ we leverage these advances to study high strain-rate plasticity through shock loading of coarse-grained copper and discuss the first non-destructive characterization of dynamic damage nucleation in the bulk.

Many experiments have studied shock loading and void formation using gas gun driven flyer plates to impact a specimen and cause spallation. ${ }^{16-26}$ These studies have shown that relative orientation of constituent grains plays a role in determining where voids form, ${ }^{19,21,22,27-29}$ that the structure of the grain boundary also plays a role ${ }^{30}$ and that the geometry of the loading direction vis-a-vis these features is also important. ${ }^{23}$ Novel modeling and simulation efforts have confirmed that grain boundaries with different character behave differently under high rate dynamic compression ${ }^{15,24}$ and demonstrated success in elucidating the void growth regime. ${ }^{31}$

In this work, we use synchrotron-based near-field High Energy Diffraction Microscopy (nf-HEDM) ${ }^{32,33}$ to map the microstructure of a $\mathrm{mm}^{3}$-sized, well-annealed copper sample. The specimen was shock loaded using an experimental setup tuned to form micro-scale voids. The shocked microstructure was then remapped, and the micro-computed tomography

a)davidmenasche@cmu.edu $(\mu \mathrm{CT})$ was performed to image the void field. All three data sets were then registered to provide fully $3 \mathrm{D}$, before and after information on the sample's orientation and void fields.

A total of 485 voids are resolved with $87 \%$ being found close to the observed grain boundaries. The distribution of void locations is non-uniform within the spall plane, implying either some asymmetry in the loading or a microstructural origin. It is found that the spalled region does have a distinct microstructure: its volume is dominated by large grains with complex topologies with a significant fraction of the volume being occupied by unusually large $\Sigma$-3 related domains $(\Sigma 3 \mathrm{D})$. These configurations do not extend to the regions where voids did not form in large numbers. A significant fraction of the voids are close to boundaries within the $\Sigma$ 3Ds. Thus, voids may be preferentially nucleated on boundaries of large grains or they may tend to occur within $\Sigma$ 3Ds. Within the somewhat skewed statistics of the spalled region, there is no statistically significant preference for the voids to form on any particular type of boundary. We conclude that systematic, non-destructive, before and after measurements hold the possibility of sorting out these questions and that it is now possible to perform dynamic loading on fully characterized microstructures so that, for example, one can avoid including or purposely include exceptional microstructural populations in the region to be spalled.

\section{EXPERIMENTAL METHODS}

A half-hard plate of $99.997 \%$ pure polycrystalline oxygen free electrolytic C101 copper was machined and annealed in vacuum at $450^{\circ} \mathrm{C}$ for $30 \mathrm{~min}$. Bingert et al ${ }^{25}$ 
(a)

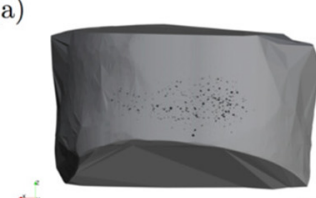

(e) (b)

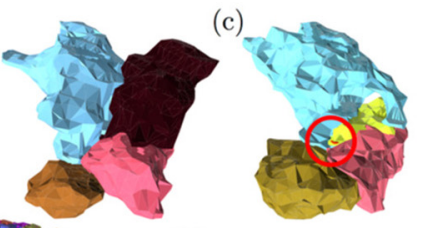

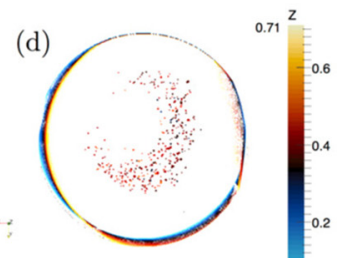

(f)

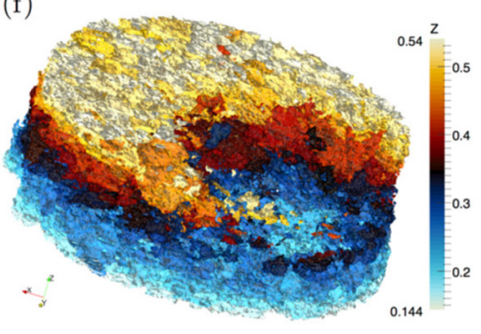

FIG. 1. A summary of the experimental data collected. (a) A cutaway view of the $\mu$ CT measured post-shocked sample. The voids are shown at an actual size in black near the mid-plane. (b) A four-grain system from the pre-shocked state, colored by mapping Rodrigues' orientation vectors to RGB values. (c) The same four-grain system extracted from the registered post-shocked state. Color differences illustrate bulk lattice rotations of the constituent grains. In the case of the maroon grain of (b), this lattice rotation crosses a periodic boundary condition within the color map producing the yellow grain of (c). Highlighted with the red circle is a void formed at the $M=4$ node connecting the four grains extracted. (d) A top down view of the sample edges segmented from $\mu$ CT of the post-shock sample includes the spall-void field created by the flyer plate impact. Voids lie in a crescent-shaped region and are slightly enlarged for visibility. Colors represent the $z$-coordinate normal to the figure. (e) A cutaway view of the pre-shocked reconstructed orientation field, colored by orientation. In (f), the reconstructed post-shocked sample, with grains colored by center of mass distance along the loading direction ( $\hat{z})$. The missing region in the right foreground is due to severe plastic deformation which prevents reliable reconstruction, though material does exist here.

performed electron backscatter diffraction (EBSD) microscopy on a characteristic free surface of the material and determined the mean grain size to be approximately $30 \mu \mathrm{m}$, excluding annealing twins. Including these features resulted in a mean grain size of $14 \mu \mathrm{m}$, though the authors note large variation in the grain sizes with some grains as large as $100-200 \mu \mathrm{m}$. A subvolume of the $1.2 \mathrm{~mm}^{2} \times 2.8 \mathrm{~mm}$ cylindrical sample's microstructure was mapped using nf-HEDM at Sector 1-ID at Argonne National Laboratory's Advanced Photon Source (APS 1-ID). The sample was illuminated with a monochromatic $65.384 \mathrm{keV}$ X-ray beam plane-focused to approximately $1.4 \mathrm{~mm} \times 2 \mu \mathrm{m}$. Bragg-diffracted beams were collected on a CCD detector optically coupled to a thin scintillation screen by rotating the sample about its axis of symmetry. Volumetric data were acquired by translating the sample parallel to its symmetry axis such that successive planar cross sections were illuminated. Images corresponding to 170 layers spaced at $4 \mu \mathrm{m}$ were collected for a mapped volume of $\approx 0.8 \mathrm{~mm}^{3}$.

The measured volume was micromachined out of the sample and embedded in a copper target assembly. Radial momentum trapping rings fabricated from the same material were utilized to prevent lateral release processes from affecting the uniaxial loading state. This assembly was shock loaded at $300 \cdot \mathrm{m} \mathrm{s}^{-1}$ with a copper impact plate $360 \mu \mathrm{m}$ in thickness, using a modified $7.6 \mathrm{~mm}$-diameter gas-driven Taylor gun. ${ }^{25}$ After shock loading, soft capture of the sample was employed to mitigate further damage. The shocked sample was remapped at APS 1-ID, where 136 layers of diffraction were collected in the manner previously described. This $1.2 \mathrm{~mm}^{2} \times 544 \mu \mathrm{m}$ volume was centered around the anticipated spall plane of the specimen, and included both highly damaged and less damaged regions. While certain especially damaged, regions were not completely reconstructable (see Fig. 1(f)), sufficient volume from the less damaged region was reconstructed so as to allow for registration of the two datasets and examination of microstructural evolution. In regions with the most damage, based on comparison with prior quasi-static measurements on copper, ${ }^{10}$ we estimate inhomogeneous local plastic strains on the order of $10 \%-20 \%$.

Parallel-beam absorption $\mu \mathrm{CT}$ images were collected to image the void field within the post-shocked sample. Using $65.384 \mathrm{keV} \mathrm{X}$-rays, the sample was illuminated with an unfocused beam of dimension $1.5 \mathrm{~mm} \times 1 \mathrm{~mm}$. The sample was fully rotated about its axis of symmetry, and radiographs were collected every $0.1^{\circ}$. These radiographs were reconstructed with the GridRec algorithm to yield 625 crosssectional images at $1.48 \mu \mathrm{m}$ resolution. ${ }^{34,35}$

\section{DATA ANALYSIS AND RESULTS}

Post-processing of the reconstructed $\mu \mathrm{CT}$ data was required to segment the void field. The $3 \mathrm{D}$ image stack represents the material density in $1.7 \times 10^{8}$ volume elements (voxels) within a $0.544 \mathrm{~mm}^{3}$ volume. Voids are recognized as connected voxels with reduced density compared to that of the fully dense material. An intensity threshold was applied to extract these features. To remove the reconstruction artifacts, these segmented regions were examined for connectivity and accepted if they were independently reconstructed in adjacent cross-sections. Lastly, any remaining reconstruction artifacts, e.g., rings from hot or dead detector pixels, were manually removed. Following void extraction, 485 unique voids were found with mean diameter $\bar{d}_{\text {void }}=4.4 \mu \mathrm{m}$. The void field extracted was then transformed into the pre-shocked reference frame, as discussed below.

To reconstruct the nf-HEDM data, the IceNine program $^{36}$ was used to optimize peak overlap between simulated diffraction from reconstruction space sample voxels and the 
observed diffraction. ${ }^{33}$ Reconstructed voxels from each plane were integrated into $3 \mathrm{D}$ volumes representing the preand post-shocked orientation fields. These states were reconstructed using equilateral triangular voxels of sidewidth $5.6 \mu \mathrm{m}$ and $2.8 \mu \mathrm{m}$, respectively. Features of interest including grains, grain boundaries, $N$-lines (lines connecting $N \geq 3$ distinct grains), and $M$-nodes (points connecting $M \geq 4$ grains) were then extracted directly from each volume; three or more contiguous voxels with crystallographic misorientation $\leq 5^{\circ}$ were defined as grains, only $N=3$ (triple) lines were segmented, and $M$-nodes were defined to be points where two or more triple lines terminated within the resolution of one voxel. Average grain orientations are computed over each set of associated voxels.

To analyze the pre-shocked state, a $1.2 \mathrm{~mm}^{2} \times 420 \mu \mathrm{m}$ region of interest (ROI) was extracted from the total of $1.2 \mathrm{~mm}^{2} \times 680 \mu \mathrm{m}$ measured. From this ROI, 16,996 grains were segmented with mean and standard deviation sphereequivalent diameter $\bar{d}_{\text {pre }}=23 \pm 20 \mu \mathrm{m}$. Many were significantly larger, and in fact, the largest 100 grains account for $25 \%$ of the ROI volume; the largest 1000 grains account for over $90 \%$. Comparison of the reconstructed pre-shocked microstructure with the EBSD image of the same sample (though not the same region) in Bingert et al. ${ }^{25}$ reveals the absence of certain thin, flat grains in the nf-HEDM reconstruction. Identified as the face centered cubic (FCC) annealing twins, these grains have misorientation $60^{\circ}\langle 111\rangle$ (coincident site lattice (CSL) boundary $\Sigma-3^{37}$ ) and share $\sim 1 / 3$ of the same Bragg peaks. For cases where the grain boundary inclination plane is also $\langle 111\rangle$, the boundary is called a coherent twin. The ability to differentiate parent and embedded twins depends on optimal experimental conditions; in this case, during collection of the pre-shocked diffraction images, conditions were compromised by beam instability. Combined with sub-optimal beam-block placement which partially attenuated diffracted beams with small Bragg angles, this prevents the resolution of small twin features. (For an illustration in which nf-HEDM is used successfully to track annealing twins, see Lin et al. $^{38}$ )

A $1.2 \mathrm{~mm}^{2} \times 400 \mu \mathrm{m}$ ROI was extracted from the postshock measurement, with 26269 grains of $\bar{d}_{\text {post }}=13 \pm 15 \mu \mathrm{m}$. The large standard deviation reflects the shape of the size distribution, which again has large positive skew. The difference in the number of grains found before and after shock treatment is ascribed to the combination of grain-breakup events and a resolution effect associated with reconstructing the post-shock volume with smaller voxels. Both the pre- and post-shocked ROIs are centered around the spall plane of the post-shocked sample state and correspond largely to the same sample volume.

A registration procedure was followed to map the two data sets into one reference frame. The optimal registration was obtained by minimizing integrated misorientation angle ${ }^{6,39}$ under rigid body rotations and translations. Higher order corrections to this registration could be computed on a grain-by-grain basis by allowing optimization within the full affine space. While this additional procedure would account for local shape change due to grain plasticity, the initial registration produced sufficient correspondence between states that further refinement was not necessary. Both the post-shock HEDM and the voids were transformed in the same way into the reference frame of the pre-shock HEDM data set, and the void field was compared to this data set. The void field was not used in determining the registration of states.

The distribution of voids along the loading direction is centered at the sample mid-plane. Looking down the loading axis, as in Fig. 1(d), while voids are concentrated near a single radius, the azimuthal distribution is crescent shaped. We discuss the possible causes for the following. The shock compression caused some lateral expansion of the sample, as shown in Fig. 1(a), a cutaway view of the $\mu \mathrm{CT}$ volume. Voids are small and sparse in the average sense: the porosity over the volume of the sample is $\sim 0.006 \%$.

Most voids nucleate on interfaces, namely, grain boundaries, triple lines, and $M$-nodes. As seen in Table I, 68\% of voids form within one voxel of these interfaces. This is as expected from previous work..$^{19,20,22,23,26,28,40}$ About $19 \%$ of voids form between voxel resolution and $10 \mu \mathrm{m}$ from one of these interfaces; these voids may well have formed at boundaries that moved during the dynamic deformation and, therefore, are not precisely at pre-shock boundary locations. Some voids $(13 \%)$ appear to form within grains $(\geq 10 \mu \mathrm{m}$ from an interface). This finding has precedent, ${ }^{27}$ but may also be the result of unresolved twins.

\section{DISCUSSION}

\section{A. Microstructural environment}

Fig. 2 shows number weighted histograms of the cross boundary lattice misorientation angle between neighboring grains before and after shock loading. Examination of the axes of misorientation in these distributions reveals that the sharp peaks at $60^{\circ}, 39^{\circ}, 35^{\circ}$, and $32^{\circ}$ correspond to the "special" CSL boundaries $\Sigma-3, \Sigma-9\left(38.9^{\circ}\langle 110\rangle\right), \Sigma-27 \beta$ $\left(35.4^{\circ}\langle 210\rangle\right)$, and $\Sigma-27 \alpha\left(31.6^{\circ}\langle 110\rangle\right)$, respectively. The widths of these peaks are broadened considerably in the post-shocked state. This finding is consistent with the average orientation of each grain being individually perturbed by the shock loading. It is also observed that the dispersion of orientations within grains increases significantly after the shock, much as is observed in quasi-static loading measurements. ${ }^{8}$

There are some differences in the microstructure local to the voided region relative to the unvoided regions of microstructure. A subregion of the ROI was used to evaluate these

TABLE I. Summary of void locations relative to the pre-shocked microstructure. Voids in one category do not appear in another.

\begin{tabular}{lc}
\hline \hline Void locations & Abundance \\
\hline Within resolution of $\mathrm{M} \geq 4$ node & $10 / 485 \approx 2 \%$ \\
Within resolution of a triple line & $126 / 485 \approx 26 \%$ \\
Within resolution of a boundary & $196 / 485 \approx 40 \%$ \\
$\leq 10 \mu \mathrm{m}$ from an interface & $91 / 485 \approx 19 \%$ \\
$>10 \mu \mathrm{m}$ from an interface & $62 / 485 \approx 13 \%$ \\
Total & $485 / 485=100 \%$ \\
\hline \hline
\end{tabular}


differences by first forming a Laplacian surface that enclosed all the voids, then expanding this surface isotropically by $100 \mu \mathrm{m}$. The resultant crescent-shaped mask partitions points within the ROI into nominally voided and nonvoided regions, with the voided region occupying the region within the mask. Grains within this voided region form a $\Sigma-3$ related domain that blankets the void field. Using three large void-hosting grains as seeds, we examine grains connected to these seeds by $\Sigma-3$ boundaries and expand to their $\Sigma-3$ connected neighbors. To limit the growth of this $\Sigma 3 \mathrm{D}$, connected grains must be members of the largest 500 grains within the ROI. The resulting domains contain 46 grains, make up $10 \%$ of the ROI, and span the void field. These large domains are characteristic of the voided region. Relative to outside, the voided region contains twice the boundary area fraction of $\Sigma-3$ connected grains belonging to domains with $\geq 13$ constituents. The mean domain size for the region outside the voided region is $6.7 \sum-3$ connected neighbors, whereas within the voided region, it is 9.0. These differences are highlighted in Fig. 3.

\section{B. Void location statistics}

The $N_{v}=196$ voids on grain boundaries are on boundaries of only 112 unique grains, which are in general large, together comprising $25 \%$ of the pre-shock ROI. The misorientation angles of these boundaries are shown in the inset of Fig. 2 in purple; in green, the boundary-area weighted misorientation distribution of all boundaries in the voided region is shown for comparison. Of interest is how the probability of void formation, $P[v]$, depends on a particular misorientation angle, $\Delta g$. The measured misorientation distribution in purple in Fig. 2 is the probability of observing a particular misorientation, given that that boundary has a void, $P[\Delta g \mid v]$. Application of Bayes' theorem allows for the reversal of the conditional

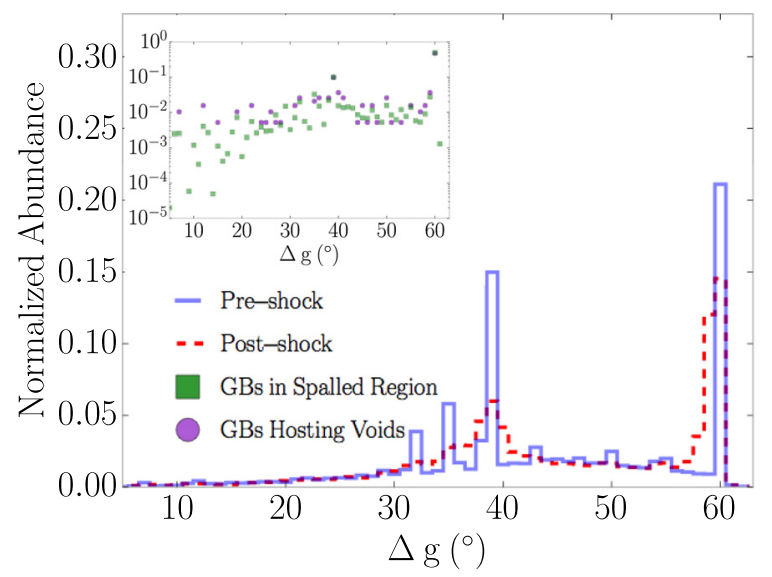

FIG. 2. Normalized, number-weighted histogram of the misorientation angle between all nearest-neighbor grains in the pre-shocked (blue) and postshocked (red) states. Histogram bins are centered on integer values between $\left[5^{\circ}, 62^{\circ}\right]$. Inset, boundary area weighted misorientation histograms for all boundaries in the spalled region (green squares) of the pre-shocked state and just those boundaries found to be associated with voids (purple circles). Histogram values are represented by the symbols and are plotted on a log scale to emphasize differences between the distributions. Bins are again centered on integer values between $\left[5^{\circ}, 62^{\circ}\right]$.

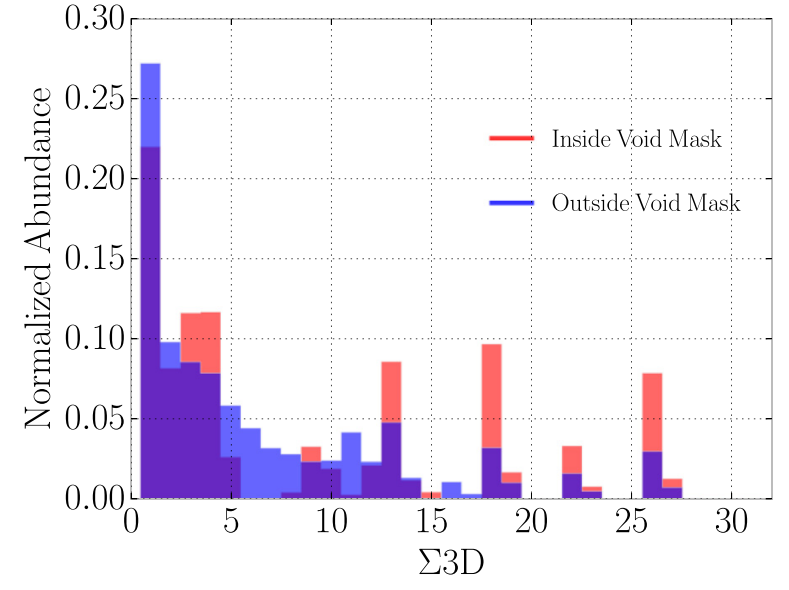

FIG. 3. Area-normalized histogram of the $\Sigma$-3 connected domain size, $\Sigma 3 \mathrm{D}$. The red distribution represents boundary area within the spalled region, while the blue distribution represents boundaries that are outside. The horizontal axis indicates the number of grains within a domain.

$$
P[v \mid \Delta g]=\frac{P[\Delta g \mid v]}{P[\Delta g]} P[v]
$$

which allows us to evaluate $P[v \mid \Delta g]$, the probability of forming a void given $\Delta g$, modulo the overall constant factor of $P[v]$ about which we are not concerned. The ratio of the distributions in the inset of Fig. 2 gives Fig. 4. Marked horizontally in yellow is the line at which $P[\Delta g \mid v] / P[\Delta g]=1$, the case of statistical independence between $v$ and $\Delta g$. The overlap of the error bars with $P[\Delta g \mid v] / P[\Delta g]=1$ indicates that there is no evidence that misorientation angle promotes or inhibits void formation among various boundary populations, special CSL or otherwise. The two peaks at the low limit of $\Delta g$ may be misleading since they are based on the formation of only two and three voids, respectively. While we have marginalized over the rotation axis and boundary inclination degrees of freedom, these are strongly coupled to $\Delta g$ for the special CSL cases. Due to the large area fraction of $\Sigma-3$ boundary within this region, the remaining $\approx 100$ voids that are on other, general high angle boundaries are not large

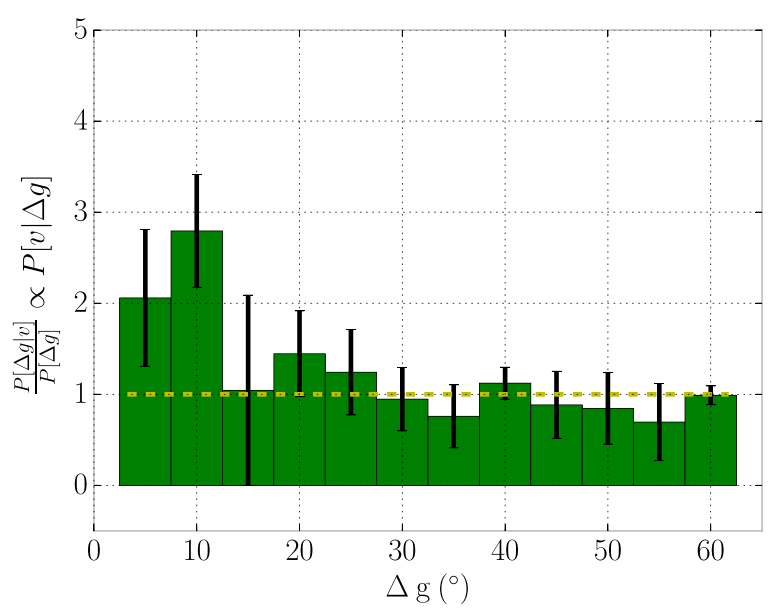

FIG. 4. Histogram proportional to $P[v \mid \Delta g]$, the probability of void formation gives a certain boundary misorientation angle, $\Delta g . N_{v}=196$. See text for details. The error bars are propagated Poisson errors from the distributions in the inset in Fig. 2. 


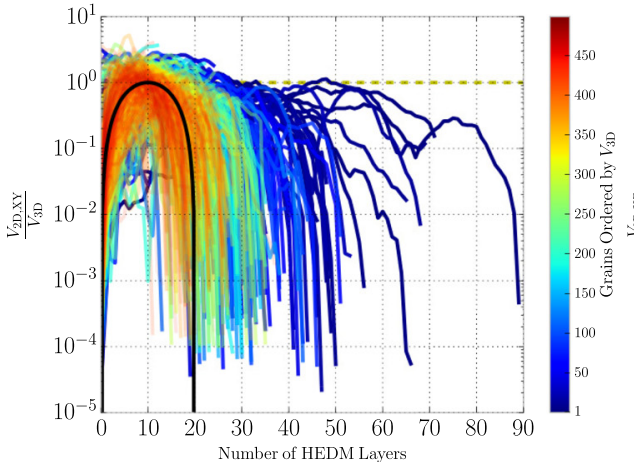

(a)

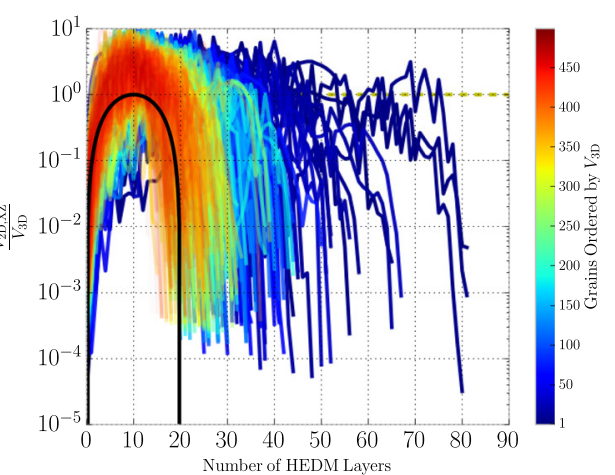

(b)

FIG. 5. Plots of the ratio between sphere equivalent volume and the actual measured volume for the largest 500 grains, ordered from largest (grain 1) to smallest (grain 500). Note that the vertical axis is scaled logarithmically. Sphere equivalent volumes are calculated by finding the sphere equivalent area of a given 2D slice in (a) the XY plane or (b) the XZ plane. Overlaid in black is the ratio plotted for a spherical grain of radius $80 \mu \mathrm{m}$. While the smallest grains are reasonably spherical, large grains are elongated; this is seen by noting the deviations from the black curve's shape in the curves corresponding to the larger grains. (Deviations between the horizontal extent of the black curve and the colored curves represent differences in grain size only.) Excepting the start and end points of each curve, regions where the computed ratio differs from unity represent cross-sections where simple stereographic estimates of grain size are misleading.

enough to statistically resolve nucleation preferences among generalized high angle boundary types.

Resolving the relative void nucleation preference between the coherent twin boundary and general $\Sigma$-3, boundary types could further validate simulations. ${ }^{15}$ For the reasons discussed above, many of the coherent grain boundaries are missing from our analysis. Utilizing a regularized smoothing algorithm to remove discretization noise ${ }^{41}$ of the $\Sigma$-3 grain boundaries observed, we calculate $<1 \%$ of the $\Sigma-3$ boundary area within the voided region is within $10^{\circ}$ of the coherence criterion; the expected fraction is substantially larger than this. ${ }^{42}$ As such, we cannot draw conclusions about nucleation preferences among this class of boundary from the current data set.

\section{Void distribution}

While the distribution of voids along the loading direction is centered at the sample mid-plane, the azimuthal distribution is nonuniform, as is clear from Fig. 1(d). This distribution may be caused by either the microstructure of the voided region itself or by loading asymmetry.

The underlying microstructure may cause this azimuthal variation. The microstructure within the voided region contains unusually large 5 3Ds comprised usually large grains. The implication is that the size of the constituent $\Sigma 3 \mathrm{D}$ may play some role in localizing the stresses that cause incipient void formation. Grain size may play a contributing role. Comparing the stresses within these regions using the farfield HEDM technique (ff-HEDM) ${ }^{43-47}$ could be illuminating.

On the other hand, some facts argue for asymmetry in the loading condition as the primary cause of this distribution. As mentioned, there is no variation in the local misorientation distribution within and outside of the voided region as would be expected if local grain boundary character explained the shape of the void field. Further, the spatial variation in void location is smooth; if there were localized microstructural features associated with spots of preferential nucleation, these features would have to be non- homogeneously distributed within the microstruture, which is unlikely for a conventionally processed specimen. Under perfectly uniaxial loading conditions, by symmetry, there should be no azimuthal strain variation from the impact. Triaxiality due to asymmetric plate impact or non-ideal lateral momentum confinement can both cause release waves which are not along the nominal loading direction. These waves, when superposed with waves along the nominal loading axis, are capable of modifying the regions of tension and compression. Notably, voids form in regions of tension, but from the data collected, one cannot determine the local strain states in the material. Indeed, mapping these states with ffHEDM could assist in the resolution of questions like these.

\section{3D grain morphology characterization}

Grains forming the $\Sigma 3$ Ds have non-convex and reentrant shapes, with protrusions that span hundreds of microns. ${ }^{48}$ The complex grain conformations and the large sizes of some of the grains within the volume cannot be inferred from planar cross sections. To show this, planar slices normal to the loading axis of the 500 largest grains in the ROI are examined. For each of these grains, a sphere equivalent volume, $V_{2 D, X Y}$, is determined using the cross-sectional area equivalent circle diameter and compared to the $3 \mathrm{D}$ measured volume from the nf-HEDM measurement, $V_{3 \mathrm{D}}$. Taking the ratio of these quantities gives an estimate for accuracy of the naive stereographic assumption. Figure 5(a) plots this quantity as a function layer index, spanning the number of $4 \mu \mathrm{m}$ spaced layers of HEDM data over which each grain persists. The black overlay represents an $80 \mu \mathrm{m}$ perfectly spherical grain, with a $V_{2 \mathrm{D} . \mathrm{XY}} / V_{3 \mathrm{D}}$ ratio of unity at the cross section at the equator of the sphere. For the largest grains, each grain's true volume is underestimated, and significant deviations from the equiaxed case show the formation of narrow protrusions from the bodies of the grains. These protrusions, however, do not appear to form isotropically; Fig. 5(b) shows the same metric computed from slices in a perpendicular plane and indicates overestimation of grain volume. Grain protrusions or long axes extend 
preferentially along the loading direction. Neither cross section accurately estimates grain volume for the larger grains in the measured region. Smaller grains exhibit apparent volume estimates more consistent with equiaxed, more spherical shapes. The $\Sigma 3 \mathrm{D}$ connected grains within the voided region are large (46 of these grains comprise $10 \%$ of the volume of the entire $\sim 1.7 \times 10^{5}$ grain ROI) and include the grains with these extending protrusions. These re-entrant shapes may also play some contributory role in void formation.

\section{CONCLUSION}

This work demonstrates a new methodology for studying shock loading that allows 3D, before and after measurements. Void observations are interpreted in the context of the surrounding initial state microstructure, i.e., the microstructure that received the shock. Such a pre-shock measurement was not possible prior to the development of HEDM and related high energy $\mathrm{X}$-ray grain mapping methods. Three dimensional information is found to significantly affect interpretation. For example, in the present case, large networks of $\Sigma$ 3Ds that are specific to the voided region limit statistical statements about the grain boundary character dependence of the susceptibility to void formation. On the other hand, the prevalence of voiding within this region raises questions as to a possible role of large grains, complex grain topologies, and/or $\Sigma 3$ Ds.

In future work, it will be possible to fully characterize samples prior to shock loading experiments and to select specific microstructural sites of interest. Adding non-destructive strain state characterization using far-field HEDM before and after loading will yield substantially more detailed information with which to test computational models. Even in the current data set, a wealth of information (e.g., grain rotation, break-up, and internal misorientation development) remains to be extracted. Avenues for further work on the current data set include analyses of changes in grain morphology and of intragranular damage accumulation through observed orientation variations. More broadly, the methodology can be employed to study myriad shock loading scenarios and specimens.

\section{ACKNOWLEDGMENTS}

The authors would like to thank many individuals for their assistance: C. M. Hefferan and R. Pokharel (CMU) for assisting with HEDM measurements at APS, S. Maddali (CMU) for boundary smoothing code, M. Prijatelj (CMU) for image artifact removal, R. Randolph (LANL) for micromachining, M. F. Lopez (LANL) for heat treating, G. T. Gray III (LANL) for providing material, M. Tucker (LANL) for APS assistance, D. Martinez (LANL) for experimentation, A. D. Rollett (CMU), R. A. Lebensohn (LANL), and C. A. Bronkhorst (LANL) for useful discussions, and E. K. Cerreta and C. A. Bronkhorst whose collaborations helped secure APS beamtime. Work at CMU was supported by LANL's Laboratory-Directed Research and Development-Directed Research (LDRD-DR Project No. 20140114DR "Mesoscale Materials Science of Ductile Damage in 4 Dimensions"). J.F.B. acknowledges the support of the Joint DoD/DOE Munitions Program and the National
Nuclear Security Administration's Science Campaign 2. Use of the Advanced Photon Source, an Office of Science User Facility operated for the U.S. Department of Energy (DOE) Office of Science by Argonne National Laboratory, was supported by the U.S. DOE under Contract No. DEAC02$06 \mathrm{CH} 11357$.

${ }^{1}$ T. Mashimo, Y. Hanaoka, and K. Nagayama, J. Appl. Phys. 63, 327 (1988). ${ }^{2}$ D. Jia, Y. M. Wang, K. T. Ramesh, E. Ma, Y. T. Zhu, and R. Z. Valiev, Appl. Phys. Lett. 79, 611 (2001).

${ }^{3}$ Q. Wei, D. Jia, K. T. Ramesh, and E. Ma, Appl. Phys. Lett. 81, 1240 (2002).

${ }^{4}$ S. R. Kalidindi, A. Bhattacharyya, and R. D. Doherty, Proc. R. Soc. London A 460, 1935 (2004).

${ }^{5}$ R. A. Lebensohn, Y. Liu, and P. P. Castaneda, Acta Mater. 52, 5347 (2004).

${ }^{6}$ R. A. Lebensohn, R. Brenner, O. Castelnau, and A. D. Rollett, Acta Mater. 56, 3914 (2008).

${ }^{7}$ A. D. Rollett, R. A. Lebensohn, M. Groeber, Y. Choi, J. Li, and G. S. Rohrer, Modell. Simul. Mater. Sci. Eng. 18, 074005 (2010).

${ }^{8}$ R. Pokharel, J. Lind, A. K. Kanjarla, R. A. Lebensohn, S. F. Li, P. Kenesei, R. M. Suter, and A. D. Rollett, Annu. Rev. Conden. Matter Phys. 5, 317 (2014).

${ }^{9}$ J. Lind, S. F. Li, R. Pokharel, U. Lienert, A. D. Rollett, and R. M. Suter, Acta Mater. 76, 213 (2014).

${ }^{10}$ R. Pokharel, J. Lind, S. F. Li, P. Kenesei, R. A. Lebensohn, R. M. Suter, and A. D. Rollett, Int. J. Plast. 67, 217 (2015).

${ }^{11}$ E. T. Seppälä, J. Belak, and R. E. Rudd, Phys. Rev. B 69, 134101 (2004).

${ }^{12}$ S. Case and Y. Horie, AIP Conf. Proc. 845, 307 (2006).

${ }^{13}$ S.-N. Luo, T. C. Germann, D. L. Tonks, and Q. An, J. Appl. Phys. 108, 093526 (2010).

${ }^{14}$ Q. An, W. Z. Han, S. N. Luo, T. C. Germann, D. L. Tonks, and W. A. Goddard, J. Appl. Phys. 111, 053525 (2012).

${ }^{15}$ S. J. Fensin, E. K. Cerreta, G. T. Gray III, and S. M. Valone, Sci. Rep. 4, 5461 (2014).

${ }^{16}$ A. Mishra, M. Martin, N. Thadhani, B. Kad, E. Kenik, and M. Meyers, Acta Mater. 56, 2770 (2008).

${ }^{17}$ J. F. Bingert, V. Livescu, and E. K. Cerreta, in Electron Backscatter Diffraction in Materials Science, edited by A. J. Schwartz, M. Kumar, B. L. Adams, and D. P. Field, 2nd ed. (Springer, 2009), Chap. 22.

${ }^{18}$ J. N. Johnson, G. T. Gray III, and N. K. Bourne, J. Appl. Phys. 86, 4892 (1999).

${ }^{19}$ L. Wayne, K. Krishnan, S. DiGiacomo, N. Kovvali, P. Peralta, S. Luo, S. Greenfield, D. Byler, D. Paisley, K. McClellan, A. Koskelo, and R. Dickerson, Scr. Mater. 63, 1065 (2010).

${ }^{20}$ D. Dennis-Koller, J. P. Escobedo-Diaz, E. K. Cerreta, C. A. Bronkhorst, B. Hansen, R. A. Lebensohn, H. Mourad, B. M. Patterson, and D. Tonks, in APS Shock Compression of Condensed Matter Meeting Abstracts (2011), p. 5005.

${ }^{21}$ J. P. Escobedo, E. N. Brown, C. P. Trujillo, E. K. Cerreta, and G. T. Gray III, J. Appl. Phys. 113, 103506 (2013).

${ }^{22}$ J. P. Escobedo, E. K. Cerreta, D. Dennis-Koller, C. P. Trujillo, and C. A. Bronkhorst, Philos. Mag. 93, 833 (2013).

${ }^{23}$ S. J. Fensin, J. P. Escobedo-Diaz, C. Brandl, E. K. Cerreta, G. T. Gray III, T. C. Germann, and S. M. Valone, Acta Mater. 64, 113 (2014).

${ }^{24}$ W. Han, E. Cerreta, N. Mara, I. Beyerlein, J. Carpenter, S. Zheng, C. Trujillo, P. Dickerson, and A. Misra, Acta Mater. 63, 150 (2014).

${ }^{25}$ J. F. Bingert, R. M. Suter, J. Lind, S. F. Li, R. Pokharel, and C. P. Trujillo, in Dynamic Behavior of Materials, Proceedings of the 2013 Annual Conference on Experimental and Applied Mechanics, edited by B. Song, D. Casem, and J. Kimberley (Springer, 2014) Chap. 48, Vol. 1.

${ }^{26}$ R. Abbasi, K. Dzieciol, and A. Borbély, Mater. Sci. Technol. 31, 540 (2015).

${ }^{27}$ A. G. Perez-Bergquist, E. K. Cerreta, C. P. Trujillo, F. Cao, and G. T. Gray III, Scr. Mater. 65, 1069 (2011).

${ }^{28}$ E. K. Cerreta, J. P. Escobedo, A. G. Perez-Bergquist, D. Dennis-Koller, C. P. Trujillo, G. T. Gray III, C. Brandl, and T. C. Germann, Scr. Mater. 66, 638 (2012).

${ }^{29}$ Y. Yang, P. Zhi-qiang, C. Xing-zhi, G. Zhao-liang, T. Tie-gang, H. Haibo, and Z. Qing-ming, Mater. Sci. Eng., A 651, 636 (2016).

${ }^{30}$ A. G. Perez-Bergquist, J. P. Escobedo, C. P. Trujillo, E. K. Cerreta, G. T. Gray III, C. Brandl, and T. C. Germann, AIP Conf. Proc. 1426, 1359 (2012). 
${ }^{31}$ R. A. Lebensohn and R. Pokharel, JOM 66, 437 (2014).

${ }^{32}$ H. F. Poulsen, Three-Dimensional X-Ray Diffraction Microscopy: Mapping Polycrystals and their Dynamics, edited by K. G. Hoeler (Springer Tracts in Modern Physics, 2004).

${ }^{33}$ S. F. Li and R. M. Suter, J. Appl. Crystallogr. 46, 512 (2013).

${ }^{34}$ Y. Wang, F. De Carlo, I. Foster, C. Kesselman, P. Lane, G. vo. Laszewski, D. Mancini, I. McNulty, M. Su, and B. Tieman, in SPIE Proc. 3772, 318 (1999).

${ }^{35}$ B. Dowd, G. Campbell, R. Marr, V. Nagarkar, S. Tipnis, L. Axe, and D. Siddons, SPIE Proc. 3772, 224 (1999).

${ }^{36}$ S. F. Li, https://github.com/FrankieLi/IceNine for "IceNine."

${ }^{37}$ D. Brandon, Acta Metall. 14, 1479 (1966).

${ }^{38}$ B. Lin, Y. Jin, C. Hefferan, S. Li, J. Lind, R. Suter, M. Bernacki, N. Bozzolo, A. Rollett, and G. Rohrer, Acta Mater. 99, 63 (2015).

${ }^{39}$ S. F. Li, J. Lind, C. M. Hefferan, R. Pokharel, U. Lienert, A. D. Rollett, and R. M. Suter, J. Appl. Crystallogr. 45, 1098 (2012).

${ }^{40}$ J. P. Escobedo, D. Dennis-Koller, E. K. Cerreta, B. M. Patterson, C. A. Bronkhorst, B. L. Hansen, D. Tonks, and R. A. Lebensohn, J. Appl. Phys. 110, 033513 (2011).
${ }^{41}$ S. Maddali and R. Suter, e-print arXiv:1601.04699.

${ }^{42}$ E. A. Holm, G. S. Rohrer, S. M. Foiles, A. D. Rollett, H. M. Miller, and D. L. Olmsted, Acta Mater. 59, 5250 (2011).

${ }^{43}$ U. Lienert, S. F. Li, C. M. Hefferan, J. Lind, R. M. Suter, J. V. Bernier, N. R. Barton, M. C. Brandes, M. J. Mills, M. P. Miller, B. Jakobsen, and W. Pantleon, JOM 63, 70 (2011).

${ }^{44}$ J. V. Bernier, N. R. Barton, U. Lienert, and M. P. Miller, J. Strain Anal. Eng. Des. 46, 527 (2011).

${ }^{45}$ J. Oddershede, B. Camin, S. Schmidt, L. P. Mikkelsen, H. O. Soerensen, U. Lienert, H. F. Poulsen, and W. Reimers, Acta Mater. 60, 3570 (2012).

${ }^{46}$ A. Beaudoin, M. Obstalecki, W. Tayon, M. Hernquist, R. Mudrock, P. Kenesei, and U. Lienert, Acta Mater. 61, 3456 (2013).

${ }^{47}$ J. C. Schuren, P. A. Shade, J. V. Bernier, S. F. Li, B. Blank, J. Lind, P. Kenesei, U. Lienert, R. M. Suter, T. J. Turner, D. M. Dimiduk, and J. Almer, Curr. Opin. Solid State Mater. Sci. 19, 235 (2014).

${ }^{48}$ B. Reed, B. Adams, J. Bernier, C. Hefferan, A. Henrie, S. Li, J. Lind, R. Suter, and M. Kumar, Acta Mater. 60, 2999 (2012). 